\title{
Synthesis of Drug-PLGA-AuNP nanomaterial for drug delivery system
}

\author{
Sourabh Mehta ${ }^{1 *}$ Jayeeta $^{2}$, V Shastri ${ }^{2}$ and H. Muthurajan ${ }^{1}$ \\ ${ }^{1}$ National Centre for Nanosciences and Nanotechnology, University of Mumbai, Vidyanagari \\ Santacruz East Mumbai-400 098, India \\ ${ }^{2}$ Murli Krishna Pharma Private Ltd.,Pune,India \\ *Email: msourabhmehta@gmail.com
}

Metallic nanoparticle has unique application in drug delivery system along with well-known application in electronics and textile industries. Gold nanoparticles (AuNP) have got special attention because of its remarkable photothermal effect. Whereas, poly (lactic-coglycolic acid) (PLGA) known for its biocompatibility and being completely biodegradable. Thus in this study we synthesized novel drug delivery system using Drug-PLGA-AuNP.

We synthesized Drug-PLGA nanoparticles using High Pressure Homogenization, making the method rapid. The process of High-pressure homogenization helped to nanoencapsulate the drug inside the polymer matrix, also increasing its bioavailability. We obtained up to $250 \mathrm{~nm}$ sized spherical polymeric particles using High pressure Homogenization (HPH) method.

The gold nanoparticles help in bio-imaging and tracking of the drug to its target receptors. We synthesized $10 \mathrm{~nm}$ sized and spherical shaped gold nanoparticles using tryptone as reducing agent and stabilizing agent. The synthesized nanoparticles were characterized by using spectroscopic and electron microscopic techniques such as UV-Vis spectroscopy, $\mathrm{X}$ ray diffraction, Fourier transform infrared spectroscopy, Transmission electron microscopy (Figure 2).

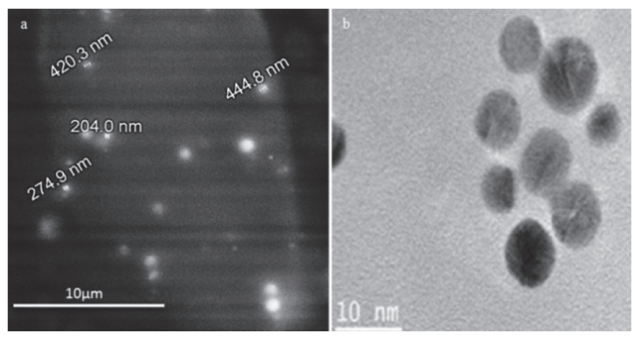

Figure 1: Electron Microscopic images a) Synthesized Drug-PLGA using HPH method; b) Tryptone stabilized AuNP
We synthesized Drug-PLGA-AuNP particles according to the flow chart of Figure 2 and characterized it using electron microscopyScanning electron microscopy, and spectroscopic method- UV Vis spectroscopy, Fourier transform infrared spectroscopy.

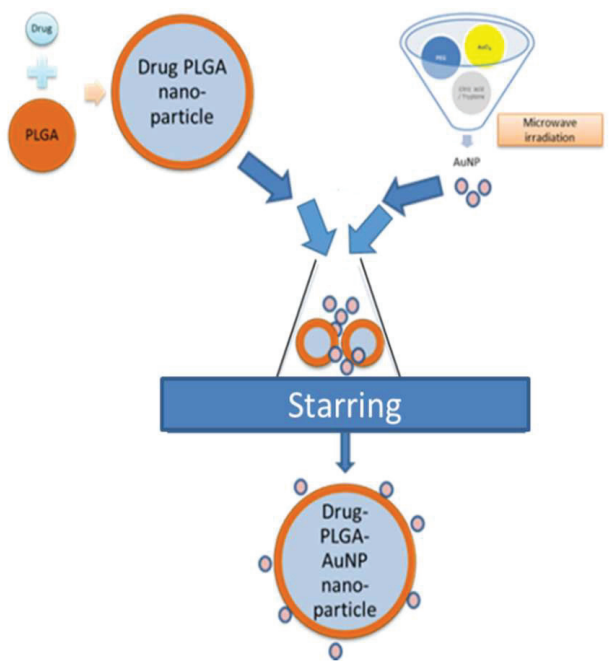

Figure 2: Schematic of synthesis of Drug-PLGAAuNP complex

Further we studied release profile of DrugPLGA-AuNP founder infra-red irradiation to learn effect of IR irradiation on drug release profile in presence of AuNP future controlled drug delivery system.

Keywords: Drug-PLGA-AuNP, drug delivery system, High-pressure homogenizer

\section{References}

1. Gabriela Romero,David J. Sanz, Yuan Qiu, Dahai Yu, et al. J. Mater. Chem. B, 2013,1, 2252-2259.

2. Nidhi Chaubey, Amaresh Kumar Sahoo, Arun Chattopadhyay, Siddhartha Sankar Ghosh. Biomater. Sci., 2014,2, 1080-1089. 